ISNN 19894090

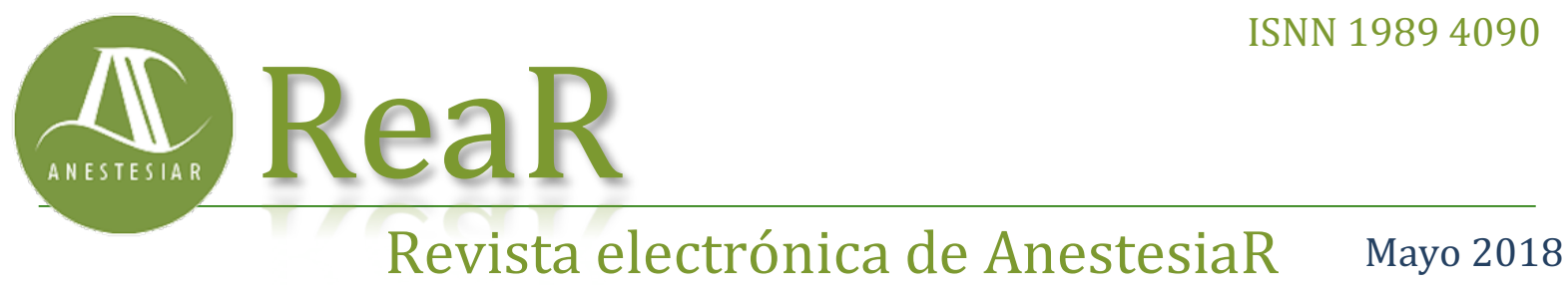

\author{
FORMACIÓN MÉDICA
}

\title{
Fluidoterapia en Neurocirugía Craneal
}

Benito Naverac H.

\section{Hospital Clínico Universitario Lozano Blesa de Zaragoza.}

\section{Resumen}

La fluidoterapia junto a la hipnosis, la protección neurovegetativa, el alivio del dolor y el mantenimiento de un adecuado bloqueo neuromuscular es uno de los pilares fundamentales de la práctica anestésica. Valorar las necesidades hídricas es un difícil desafío en el paciente neuroquirúrgico.

La bibliografía actual, va orientada a que la fluidoterapia debería tener como objetivo el mantenimiento de la homeostasis, para favorecer un equilibrio entre el aporte de líquidos y las pérdidas. Lo cual requiere una estrategia guiada por objetivos, es decir los líquidos son administrados para conseguir una mejora del transporte de oxígeno y no para aportar genéricamente la cantidad de fluido que se considere empíricamente adecuada (reglas predeterminadas fijas, regla 4.2.1).

\section{Introducción}

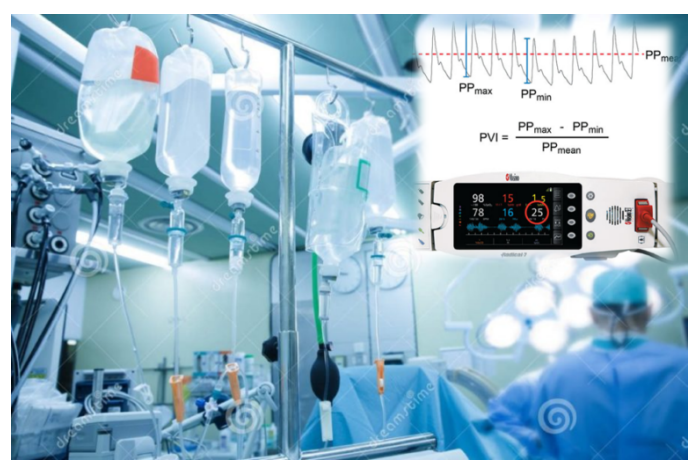

La fluidoterapia junto a la hipnosis, la protección neurovegetativa, el alivio del dolor y el mantenimiento de un adecuado bloqueo neuromuscular es uno de los pilares fundamentales de la práctica anestésica. Valorar las necesidades hídricas es un difícil desafío en el paciente neuroquirúrgico.

La bibliografía actual, va orientada a que la fluidoterapia debería tener como objetivo el mantenimiento de la homeostasis, para favorecer un equilibrio entre el aporte de líquidos y las pérdidas. Lo cual requiere una estrategia guiada por objetivos, es decir los líquidos son administrados para conseguir una mejora del transporte de oxígeno y no para aportar genéricamente la cantidad de fluido que se considere empíricamente adecuada (reglas predeterminadas fijas, regla 4.2.1).

La mayor parte de los trabajos científicos en esta área se han hecho en Cirugía Colorrectal. Existe muy poca bibliografía al respecto en Neurocirugía.
"We must use the right kind of fluid in appropriate amounts at the right time." Chappell D et al. 2008

\section{PARTICULARIDADES DE LA FLUIDOTERAPIA EN NEUROCIRUGÍA}

La necesidad de mantener una Presión Intracranial (PIC) constante hace que el paso de fluidosal intersticio neuronal deba controlarse muy estrictamente: 
El cerebro y la médula espinal están aislados del compartimento intravascular por la existencia de la Barrera Hematoencefálica. El endotelio vascular cerebral tiene unas uniones muy estrechas (tight junctions), que lo hacen impermeable al transporte pasivo de solutos, pero no de agua, la cual circula libremente según el gradiente osmótico. La osmolaridad es, por tanto, el determinante fundamental del movimiento de líquido a través de la barrera hematoencefálica.

El cerebro carece de drenaje linfático, por lo que las fuerzas hidrostáticas y osmóticas (intravasculares, intersticiales e intracelulares) están en equilibrio sin intercambio de fluido neto entre la membrana capilar y el intersticio.

\section{En el cerebro patológico el manejo de la fluidoterapia tiene algunas particularidades:}

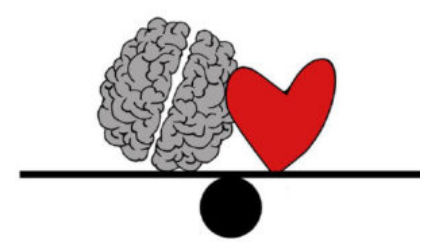

- Ruptura de la Barrera hematoencefálica y desarrollo de edema cerebral por lo que con frecuencia existe necesidad de administrar medidas que con frecuencia llevan a la aparición de deshidratación y trastornos hidroelectrolíticos (manitol, suero salino hipertónico).

- Desarrollo de trastornos tales como: Diabetes Insípida, Síndrome Pierde Sal, y Síndrome de secreción inadecuada de ADH.

- Si el aporte de líquidos es excesivo, en aquellos casos en los que la barrera hematoencefálica está rota, puede agravarse el edema cerebral mientras que si el aporte de fluido es insuficiente puede llevar a una inadecuada perfusión tisular. Por lo que la fluidoterapia en neurocirugía se basa en mantener un equilibrio estrecho entre "corazón y cerebro".

\section{RECOMENDACIONES \\ FLUIDOTERAPIA EN NEUROCIRUGÍA (Tabla 1)}

DE

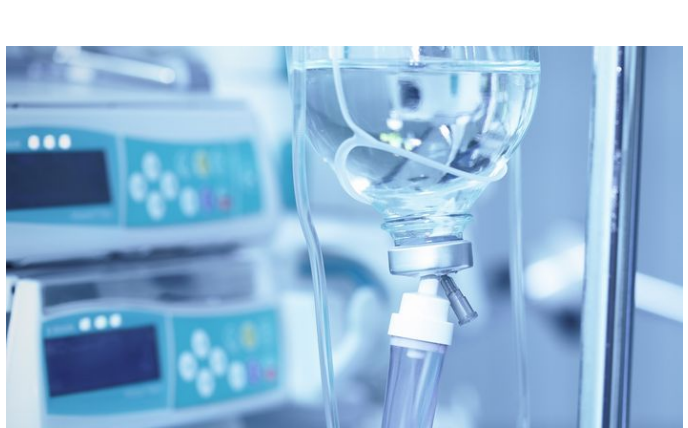

- Para reemplazar las pérdidas líquidas extracelulares secundarias a la diuresis y las pérdidas insensibles se deben utilizar Cristaloides Isotónicos (Suero Fisiológico y Plasmalyte) a una velocidad no superior a 1 $\mathrm{ml} / \mathrm{kg} /$ hora.

- Las pérdidas sanguíneas deben reemplazarse con soluciones isooncóticas. Importante mantener unos niveles de hemoglobina en sangre adecuados. Es razonable mantener unos niveles de hemoglobina en torno a 8-10 g/dl (individualizar en cada caso la cifra adecuada). Tras la ruptura de la barrera hematoencefálica, los hematíes son lo único que no abandona el capilar.

- Monitorizar la osmolaridad sérica, con el objeto de mantenerla o de elevarla ligeramente. No sobrepasar los 320 $\mathrm{mOsm} / \mathrm{L}$ por riesgo de insuficiencia renal.

- Evitar la administración de líquidos hipotónicos respecto del plasma (dextrosa al 5\%, Ringer lactato), ya que pueden agravar el edema cerebral.

- Evitar las soluciones que contengan glucosa, salvo que el paciente sea diabético, en ese caso se debe administrar glucosa e insulina para evitar la cetosis (con controles de glucosa). Está claramente demostrado que la hiperglucemia agrava el pronóstico neurológico.

- No hay indicaciones claras para la administración de coloides sintéticos en neurocirugía, hay que tener en cuenta que, los dextranos y almidones en grandes cantidades pueden ir asociados a coagulopatía. 


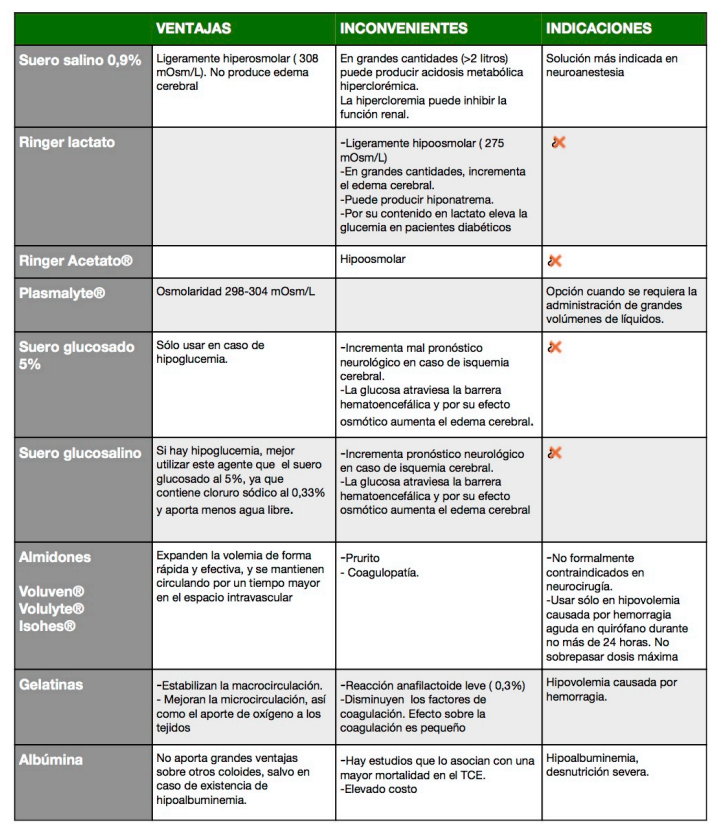

\section{FLUIDOTERAPIA GUIADA POR OBJETIVOS (FGO)}

- La fluidoterapia guiada por objetivos, tiene como finalidad mejorar la perfusión tisular y optimizar el volumen vascular mediante diversos sistemas dinámicos de monitorización hemodinámica. Esos sistemas se basan en evaluar cómo responde el sistema cardiovascular tras la administración de un aporte de líquidos a carga creciente.

- Los parámetros hemodinámicos "dinámicos ("cambios en el volumen sistólico, variación de la presión de pulso durante la ventilación a presión positiva...) han demostrado mayor exactitud para predecir el estado de la volemia del paciente y la respuesta a la expansión de volumen que los parámetros "estáticos", (Presión Aurícula Derecha, Presión Venosa Central, Presión Pulmonar Arterial Enclavada, etc), los cuales no consideran el flujo a través del sistema vascular, sino que simplemente miden componentes de presión del sistema. Es decir, estamos estimando un volumen a través de una presión, lo cual puede llevar a imprecisiones.

- Los principios en los que se basa la FGO, están relacionados con el mecanismo de Frank-Starling. Si el
Volumen Sistólico (Vs) y el gasto cardiaco aumentan tras la administración de un bolo predeterminado de líquido, se considera que al estar en la parte de inclinación ascendente de la curva (Fig.1) necesita la administración de una nueva carga de líquidos, ya que el corazón responde favorablemente a su administración.

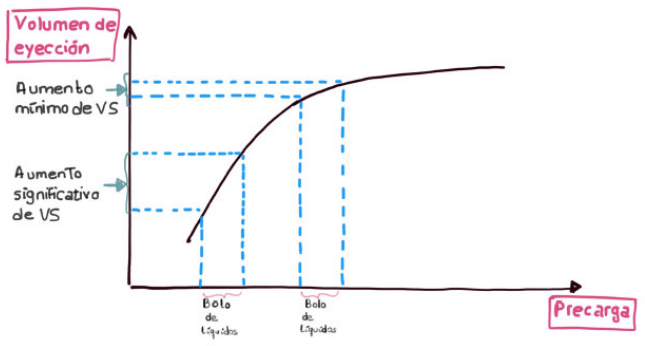

Fig 1. Mecanismo de Frank

Existen muchos métodos de monitorización hemodinámica. En la tabla.2 se indican los más comunes, los menos cruentos y los que más aplicabilidad tienen en neurocirugía.

\section{CONCLUSIONES}

El cristaloide de elección en neurocirugía es el Suero Salino al 0,9\%, ya que es el que menos edema cerebral produce. Es recomendable administrar plasmalyte ${ }^{\circledR}$ en los casos en que se tengan que administrar unos volúmenes elevados de cristaloides ( $>2$ litros). Se deben evitar los cristaloides hipotónicos (Ringer Lactato ${ }^{\circledR}$ Y Ringer Acetato () y las soluciones que contengan glucosa (salvo que exista hipoglucemia).Los coloides no están formalmente contraindicados, pero se debe valorar cuidadosamente su indicación ya que pueden producir coagulopatía cuando se administran en grandes dosis. 


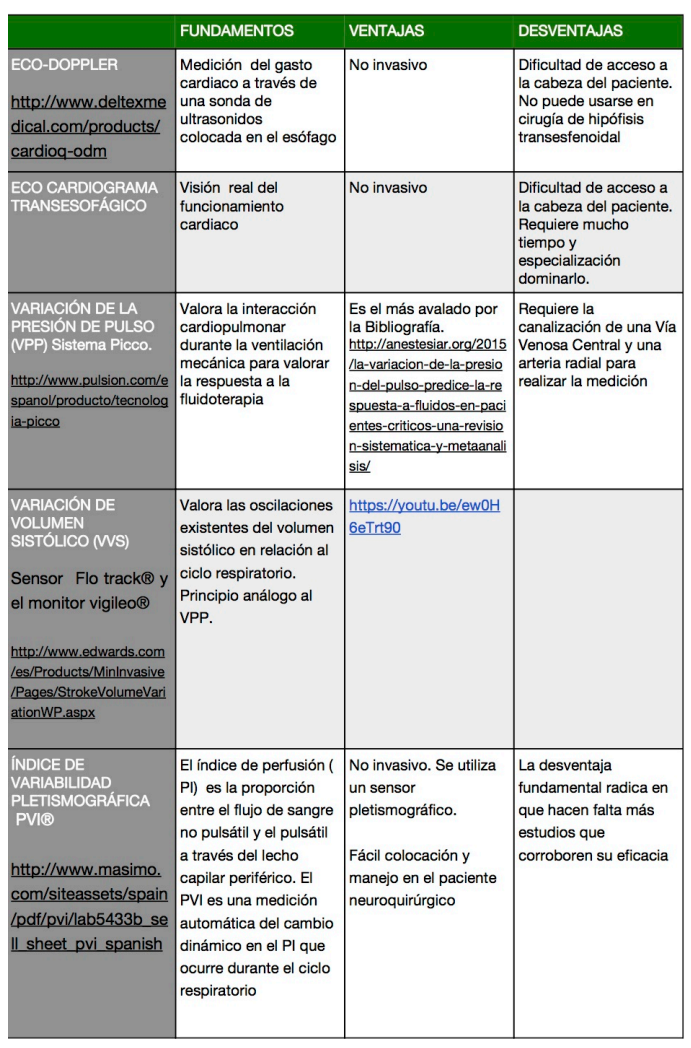

Es importante mantener la normovolemia (eso si evitando la hipoosmolaridad), para lo cual es muy útil realizar FGO. Es, por tanto, aconsejable disponer de un sistema de medición de gasto cardiaco, no invasivo, de fácil accesibilidad y manejo. Hace falta la realización de más estudios clínicos sobre FGO en neurocirugía, ya que la bibliografía al respecto es escasa.

\section{Bibliografía}

1. Singer, M., J. Clarke, and E.D. Bennett, Continuous hemodynamic monitoring by esophageal Doppler. Crit Care Med, 1989. 17(5): p. 447-52.(PubMed)

2. Deflandre E, Bonhomme V, Hans P: Delta down compared with delta pulse pressure as an indicator of volaemia during intracranial surgery, $\mathrm{Br} \quad \mathrm{J}$ Anaesth 100:245-250, 2008. (HTML)

3. Berkenstadt $\mathrm{H}$, Margalit $\mathrm{N}$, Hadani $\mathrm{M}$, et al: Stroke volume variation as a predictor of fluid responsiveness in patients undergoing brain surgery, Anesth Analg 92:984-989, 2001. (PubMed)

4. Guías clínicas para la optimización hemodinámica perioperatoria de los pacientes adultos durante la cirugía no cardiaca. Sedar 2015.

5. https://www.sedar.es/file/2015/10/Guiasclinicas-para-la-optimizacion-hemodinamicaperioperatoria-de-los-pacientes-adultos-durantela-cirugia-no-cardiaca.pdf

Correspondencia al autor

Helena Benito Naverac

helenabenito@yahoo.es

FEA de Anestesiología y Reanimación. Hospital

Clínico Universitario Lozano Blesa de Zaragoza.

Aceptado para blog en noviembre de 2017. 